\title{
Torsion of Pedunculated Subserous Myoma
}

\author{
Eliza Shrestha ${ }^{\mathrm{a}, \mathrm{c}}$, Herojit Singh Ngangbam ${ }^{\mathrm{b}}$, Yuebo Yang ${ }^{\mathrm{a}}$, Xiaomao Li ${ }^{\mathrm{a}}$
}

\begin{abstract}
We report a case of torsion of a pedunculated uterine leiomyoma. A 56-year-old married Chinese female presented at the emergency department with complaints of severe lower abdominal pain on July 27, 2010. A transabdominal sonogram revealed multiple uterine masses, including a pedunculated mass. With a preoperative diagnosis of torsion of a pedunculated subserosal leiomyoma, the patient underwent a total abdominal hysterectomy with bilateral salpingo-oophorectomy. On gross inspection, the resected myoma was ischemic, and both focal hemorrhage and necrosis were noted in the pathology report. In conclusion, torsion of a pedunculated uterine myoma is rare but represents a surgical emergency to improve symptoms and avoid consumptive coagulopathy.
\end{abstract}

Keywords: Torsion; Uterine leiomyoma; Subserous myoma

\section{Introduction}

Uterine leiomyomas are smooth muscle tumors of the uterus. They are found in women throughout the world. Leiomyomas may be submucosal, intramural, subserosal or found in the broad ligament [1]. As torsion may cause irreversible ischaemic damage to the uterus, leading to rapid clinical deterioration [2], prompt and accurate diagnosis is crucial to effective management of this potentially life-threatening condition. Occasionally, subserosal leiomyomas may be-

\section{Manuscript accepted for publication February 4, 2011}

a Department of Gynecology, The Third Affiliated Hospital of Sun Yat-sen University, Guangzhou, 510630, China

${ }^{b}$ Department of Gynecology, The Second Affiliated Hospital, Sun

Yat-sen University, Guangzhou 510120, China

cCorresponding author: elizashrestha@hotmail.com

doi:10.4021/jmc136w come pedunculated. It is even less common for pedunculated leimyomas to undergo torsion. There are only a few reported cases in the literature. We present a case of pedunculated uterine leiomyoma

\section{Case Report}

A 56-year-old married native Chinese women presented at emergency department with complain of severe lower abdominal pain on July 27, 2010. Physical Examination revealed tenderness to deep palpation as well as rebound tenderness in the right lower quadrant. Abdominal beta Ultrasound revealed enlarged uterus with multiple myoma, fluid in pelvic cavity about $31 \mathrm{~mm}$. White blood cell count was $10,770 / \mu 1$. There were no signs of gastrointestinal symptoms such as vomiting, diarrhea or constipation. No Rovsing's sign, poas sign or obturator sign were noted and, according to the progress of her symptoms, the possibility of acute appendicites was excluded by the attending surgeon. A transabdominal sonogram revealed multiple uterine masses that were consistent with multiple uterine leiomyomas. The

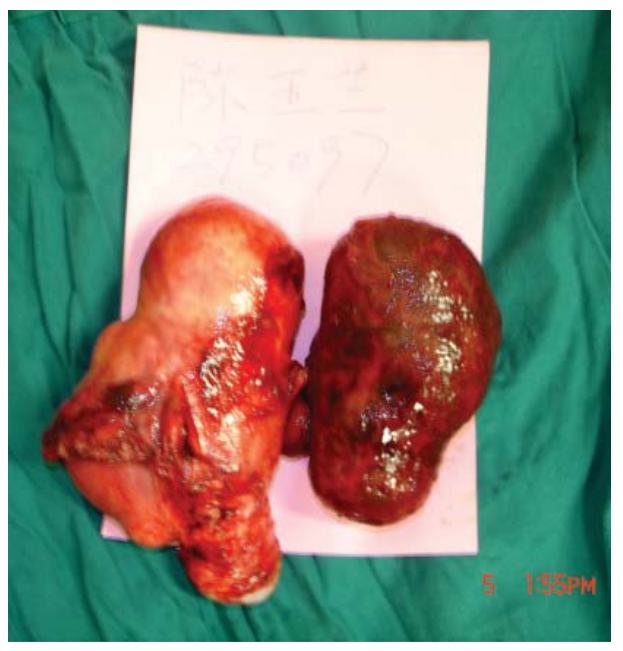

Figure 1. Pedunculated leiomyoma (bluish mass on the right side) that had undergone torsion is seen after hysterectomy. 
largest one measured $6.4 \times 6.1 \mathrm{~cm}$ and, and was located in the myometrium of the uterine fundus. There was another large mass measuring $7.6 \mathrm{~cm} \times 6.0 \mathrm{~cm}$ in size. Its pattern was homogenous and same as the myoma in the uterine fundus. We considered that it was a uterine subserosal mass in the left side of the pelvis. Given that the painful area was in the same location as the uterine subserosal mass, our preoperative diagnosis was a pedunculated uterine myoma that had undergone torsion. The patient gave consent to surgical intervention. A pedunculated leiomyomas that had undergone torsion of entire myoma beyond 360 degrees was discovered intraoperatively. The leiomyoma was approximately $5 \mathrm{~cm}$ in diameter and grossly bluish in colour (Fig. 1). An uncomplicated total abdominal hysterectomy with bilateral salpingooopehorectomy was performed. Focal haemorrhage and necrosis were noted in the pathology report. The patient had an uneventful postoperative course and was discharged on August 15, 2010.

\section{Discussion}

Torsion of a leiomyoma is very rare. The existence of a pedunculated leiomyoma is a requisite for torsion. The stalk of the pedunculated myoma must be thin and long in order for it to undergo rotation and torsion. The size of the myoma is a key factor for irreversible torsion. It is easy to correct torsion spontaneously in pedunculated leiomyomas that are small. However, large pedunculated leiomyomas are difficult to correct because there is little room to undergo torsion.

The etiology is the main factor for torsion of leiomyoma. It has a relationship between adjacent organs, such as the uterine corpus, the bowel or the pelvic side wall. After rotation of the stalk of a pedunculated leimyoma, the leimyoma may become fixed in place by surrounding organs then it becomes ischemic or necrotic, with resultant abdominal pain. Therefore consideration of a pedunculated leimyoma that has undergone torsion in the differential diagnosis is the key for early surgical intervention and treatment.

Bloch et al reported that the diagnosis of torsion of pedunculated leiomyoma could be established by magnetic resonance imaging but in the ultrasound pedicle of a subserous leiomyoma can be very thin which is frequently invisible [3]. In our case, ultrasound did not reveal leiomyoma had undergone torsion as preoperative diagnosis of torsion was based on sonographic findings as well as clinical presentation.

Roy et al reported that the torsion of uterine leiomyoma could be diagnosed by computed tomography but ultrasound is less sensitive [4]. But we were able to find the pedunculated myoma with ultrasound. However, the sonographic findings alone did not reveal that the leimyoma had undergone torsion.

The management of torsion of a uterine leiomyoma should be immediate in the case of infarction or necrosis.
In young patients who may wish to preserve fertility, operative considerations are the viability of the uterus and the patient's desire to preserve fertility. Hysterectomy should be considered in women past reproductive age $[5,6]$. Otherwise abdominal myomectomy is the most common surgical intervention. Katsumori et al described uterine artey embolization for the treatment of pedunculated leiomyoma [7]. Their cases were confirmed with baseline sagittal and axial magnetic resonance imaging. The pedunculated leiomyoma was $\geq 2 \mathrm{~cm}$ stalk diameter which found no serious complication after embolization.

Regarding the case presented here, we did not use advanced radiographic imaging techniques such as computed tomography or magnetic resonance imaging, as ultrasound is the primary imaging modality for the evaluation of females with pelvic pain. Moreover, CT and MRI are not commonly used in an emergency as the first examination to explore any type of abdominal and pelvic pain. Therefore, we felt that ultrasonography and clinical presentation were sufficient.

We conclude that early diagnosis of torsion of a uterine pedunculated myoma is important because ischemia or necrosis can cause consumptive coagulopathy or severe pain. Consumptive coagulopathy is very dangerous for the patient. Severe pain for a long time without relief is intolerable and is an indication for emergency surgery. The diagnosis can be made based on ultrasound and clinical findings. Emergency surgery must be performed as early as possible to improve symptoms and avoid consumptive coagulopathy.

\section{References}

1. Berek JS. Chapter 13: Benign Disease of the Female Reproductive Tract. In: Novak's Gynecology, 13th edition. Philadelphia: Lippincott Williams \& Wilkins, 2002:380.

2. Grover S, Sharma Y, Mittal S. Uterine torsion: a missed diagnosis in young girls? J Pediatr Adolesc Gynecol 2009;22(1):e5-8.

3. Marcotte-Bloch C, Novellas S, Buratti MS, Caramella T, Chevallier P, Bruneton JN. Torsion of a uterine leiomyoma: MRI features. Clin Imaging 2007;31(5):360-362.

4. Roy C, Bierry G, El Ghali S, Buy X, Rossini A. Acute torsion of uterine leiomyoma: CT features. Abdom Imaging 2005;30(1):120-123.

5. Nicholson WK, Coulson CC, McCoy MC, Semelka RC. Pelvic magnetic resonance imaging in the evaluation of uterine torsion. Obstet Gynecol 1995;85(5 Pt 2):888890.

6. Dua A, Fishwick K, Deverashetty B. Uterine torsion in pregnancy: a review. The Internet Journal of Gynecology and Obstetrics 2006;6(1).

7. Katsumori T, Akazawa K, Mihara T. Uterine artery embolization for pedunculated subserosal fibroids. AJR Am J Roentgenol 2005;184(2):399-402. 